\title{
Suppression of Idol expression is an additional mechanism underlying statin-induced up-regulation of hepatic LDL receptor expression
}

\author{
BIN DONG, MINHAO WU, AIQIN CAO, HAI LI and JINGWEN LIU \\ Veterans Affairs Palo Alto Health Care System, Palo Alto, CA 94304, USA
}

Received July 26, 2010; Accepted September 9, 2010

DOI: $10.3892 /$ ijmm.2010.559

\begin{abstract}
Recent studies have identified proprotein convertase subtilisin/kexin type 9 (PCSK9) and Idol as negative regulators of low density lipoprotein receptor (LDLR) protein stability. While the induction of PCSK9 transcription has been recognized as a limitation to the statin cholesterollowering efficacy at higher doses, it is unknown whether Idol is involved in the statin-mediated up-regulation of the hepatic LDLR. Here we report that statins exert opposite effects on PCSK9 and Idol gene expression in human hepatoma-derived cell lines and primary hepatocytes isolated from hamsters and rats. While PCSK9 expression was induced, the level of Idol mRNA rapidly declined in statin-treated cells in a dosedependent manner. This differs from the effect of the liver $\mathrm{X}$ receptor ligand, GW3965, which increased the expression of both PCSK9 and Idol. We further show that cellular depletion of Idol by siRNA transfection did not change PCSK9 expression levels in control and statin-treated cells; however, the basal level of LDLR protein increased by $60 \%$ in Idol siRNA transfected HepG2 cells. More importantly, the increase in LDLR protein abundance by rosuvastatin and atorvastatin treatment was compromised by Idol siRNA transfection. Collectively, our present findings suggest that the suppression of Idol gene expression in liver cells is an additional mechanism underlying the statin-induced upregulation of hepatic LDLR expression. This may contribute to the hypocholesterolemic effects of statins observed in clinical settings.
\end{abstract}

Correspondence to: Dr Jingwen Liu, Veterans Affairs Palo Alto Health Care System, 3801 Miranda Avenue, Palo Alto, CA 94304, USA

E-mail: jingwen.liu@va.gov

Abbreviations: ATV, atorvastatin; Idol, inducible degrader of the LDLR; LDL, low density lipoprotein; LDL-C, LDL-cholesterol; LDLR, LDL receptor; LPDS, lipoprotein-deficient serum; LXR, liver X receptor; PCSK9, proprotein convertase subtilisin/kexin type 9; RSV, rosuvastatin; SRE, sterol regulatory element; SREBP, SRE-binding proteins

Key words: Idol, PCSK9, LDL receptor, statins, LXR

\section{Introduction}

The concentration of low density lipoprotein-cholesterol (LDL-C) is recognized as a critical predictor of atherosclerosis and coronary heart disease (CHD) (1-3). There is abundant evidence indicating that the reduction of LDL-C lowers morbidity and mortality from CHD events (4). The activity of the LDL receptor (LDLR) in the liver is a major determinant of plasma LDL-C levels (5-7). The most successful strategy to reduce circulation levels of LDL involves the up-regulation of the LDLR activity, thereby enhancing LDL clearance from the circulation, which, in turn, slows atherosclerotic development and reduces the risk of CHD (8).

Statins are competitive inhibitors of $\mathrm{HMG}-\mathrm{CoA}$ reductase, the rate-limiting enzyme in cholesterol biosynthesis, which converts HMG-CoA to mevalonate. Statins decrease plasma cholesterol by inducing intracellular cholesterol depletion and by up-regulating the hepatic LDLR through a wellcharacterized transcriptional mechanism. LDLR transcription is predominantly controlled by the sterol regulatory element binding protein-2 (SREBP2), one of the three SREBP family members that regulate many genes involved in cholesterol and fatty acid synthesis (9-11). During cholesterol-depleted conditions, SREBP2 translocates from the cytoplasm to the nucleus where it binds to the SRE-1 element of the LDLR promoter and activates gene transcription. This leads to an increased number of LDLR on the surface of the hepatocytes, resulting in an enhanced uptake of LDL particles from the circulation. This statin-induced activation of the SREBP pathway, however, has an attenuating effect on the LDLR protein levels in the liver through a concomitant induction of proprotein convertase subtilisin/kexin type 9 (PCSK9) mediated by an SRE motif embedded in the PCSK9 promoter $(12,13)$. PCSK9 is a liver-derived plasma protein that binds to the extracellular EGF-A domain of LDLR and enhances the intracellular degradation of $\operatorname{LDLR}(14,15)$. The discovery of PCSK9 and its induction by statin treatment has changed the traditional view on how statin regulates LDLR expression. Nowadays PCSK9 is considered a limitation to the statin LDLlowering clinical efficacy at higher doses (16).

Recently, another player in the regulation of LDLR protein stability was discovered. A protein named inducible degrader of the LDLR (Idol) was found to induce ubiquitination of the LDLR on its cytoplasmic domain, which promotes LDLR degradation in the lysosomal compartment (17). Distinct 
from PCSK9, an SREBP-target gene, Idol gene expression is largely controlled by the nuclear liver X receptors (LXRs), key regulators of transcription in cholesterol metabolism. LXR $\alpha$ and LXRß are sterol-dependent nuclear receptors activated in response to cellular cholesterol excess (18). When bound to their endogenous oxysterol ligands or synthetic ligands such as GW3965 and T0901317, LXRs activate the transcription of target genes that are primarily involved in cholesterol efflux. Among these are the ATP binding cassette proteins G5, G8, and ABCA1 (19). The identification of Idol as a direct target gene of LXR revealed a new function of LXR in cholesterol uptake through its effect on modulating LDLR protein levels.

In light of this newly discovered role of Idol in LDLR protein stability and the coordinated regulation of intracellular sterols by the SREBP and LXR signaling pathways, we examined the effects of statins and the LXR agonist GW3965 on the expression of Idol and PCSK9 in human hepatomaderived cell lines and primary hepatocytes isolated from hamsters and rats. We demonstrate, for the first time, that statin treatment produced opposite effects on PCSK9 and Idol gene expression. Statins inhibit Idol mRNA expression, while up-regulating PCSK9 expression. This is distinct from the effect of the LXR ligand which increased the expression of both PCSK9 and Idol.

\section{Materials and methods}

Cells and reagents. The human hepatoma cell line HepG2 was obtained from the American Type Culture Collection and the human hepatoma-derived Huh7 cells were generously provided by Dr Ting-Ting Huang (VA Palo Alto Health Care System). HepG2 cells were cultured in Minimum Essential Medium Eagle (MEM) supplemented with $10 \%$ fetal bovine serum (FBS). Huh7 cells were cultured in Dulbecco's Modified Eagle Medium (DMEM) supplemented with 10\% FBS. Rosuvastatin (RSV) calcium was obtained from AstraZeneca (Macclesfield, UK) and atorvastatin (ATV) was purchased from AK Scientific (Mountain View, CA). GW3965 and the anti- $\beta$-actin monoclonal antibody were purchased from Sigma-Aldrich. The rabbit anti-LDLR antibody was obtained from BioVision (Mountain View, CA) and the rabbit anti-PCSK9 antibody was obtained as previously described (13). Lipoprotein deficient serum (LPDS) was purchased from HyClone (Logan, UT).

Primary hepatocyte preparation. Primary hepatocytes were prepared from male Golden Syrian hamsters and SpragueDawley rats using procedures previously described (20). Preparations with $>90 \%$ cell viability were used for further experiments. Viable hepatocytes were plated at $2 \times 10^{6}$ cells/well in 6-well BD Falcon Primaria Tissue Culture Plates in attaching medium [10\% FBS in Williams' medium $\mathrm{E}$ (Invitrogen)] and incubated at $37^{\circ} \mathrm{C}$ in a $\mathrm{CO}_{2}$ tissue culture incubator for $3 \mathrm{~h}$. Unattached cells were washed out, the culture medium was replaced with the HepatoZYME-SFM medium (Invitrogen, CA) and the cells were incubated overnight followed by the $24 \mathrm{~h}$ drug treatment.

siRNA transfection. Pooled siRNAs against Idol were purchased from Dharmacon (ID: L-006976-00). HepG2 cells were seeded in either a 6 -well plate $\left(3 \times 10^{5}\right.$ cells/well $)$ or a 12 -well plate $\left(1.5 \times 10^{5}\right.$ cells/well $)$ with MEM supplemented with $10 \%$ FBS. After the siRNA transfection of $24 \mathrm{~h}$, the cells were switched to MEM supplemented with 10\% LPDS. The next day, the cells were treated with drugs followed by total RNA or protein isolation.

RNA isolation and quantitative PCR. For HepG2 or Huh7 hepatoma cell lines, cells were seeded in 12-well cell culture plate for $24 \mathrm{~h}$ in a medium containing $10 \%$ FBS. Cells were switched to a medium containing $10 \%$ LPDS overnight before the drug treatments began. Total RNA was isolated using an RNA isolation kit from Zymo Research (Orange, CA). Real-time (RT) PCR was conducted as previously described (13). The results are averages of triplicate assays normalized to GAPDH.

The human primer sequences used were: Idol forward (cgaggactgcctcaacca) and reverse (tgcagtccaaaatagtcaacttct) (17); LDLR forward (gacgtggcgtgaacatctg), and reverse (ctggcaggcaatgctttgg); PCSK9 forward (aggggaggacatcat tggtg) and reverse (caggttgggggtcagtacc); GAPDH forward (atggggaaggtgaaggtcg) and reverse (ggggtcattgatggcaacaata).

The hamster primer sequences used were: Idol forward (cacccacaccagtcttctca) and reverse (acctggcatgtccagtaagc); LDLR forward (ctccactctatctccagcattg) and reverse (tttcagc caccaaattaacatc); PCSK9 forward (atcctcacaggcctggagtt) and reverse (ctgtgatgacctctggagca); GAPDH forward (acccagaa gactgtggatgg) and reverse (cgacatgtgagatccacgac).

The rat primer sequences used were: Idol forward (cagcga aggacagaaactcc) and reverse (gttaccggactccttggtga); LDLR forward (accgccatgaggtacgtaag) and reverse (cggcgctgtagatc tttctc); PCSK9 forward (ttagtcttcgcccagagcat) and reverse (ctcctcaggcacactgttga); GAPDH forward (agacagccgcatctt cttgt) and reverse (cttgccgtgggtagagtcat).

Western blot analysis. LDLR and PCSK9 protein expression in HepG2 and Huh7 cells was assessed by Western blot analysis as previously described (21). The specific immunoreactive bands were visualized using an ECL Plus kit (GE Healthcare Life Sciences, Piscataway, NJ) and quantified with the Kodak Molecular Imaging Software (Kodak, New Haven, CT).

Statistical analysis. All results are representative of between two to four separate experiments. Real-time PCR data and densitometry are expressed as mean \pm SEM. Statistical analysis was performed using a two-tailed Student's t-test or a one-way ANOVA followed by the Bonferroni post test. A probability value of $\mathrm{p}<0.05$ was considered statistically significant.

\section{Results}

Statins exert opposite effects on mRNA expression of Idol and PCSK9. To examine the involvement of Idol in statinmediated up-regulation of the LDLR, a time-dependent effect of RSV on the mRNA expression of Idol, PCSK9, and LDLR was examined by RT-PCR. HepG2 and Huh7 cells were treated with RSV at a dose of $5 \mu \mathrm{M}$ for varying lengths of time. Fig. 1A shows that the amount of Idol mRNA in HepG2 
A

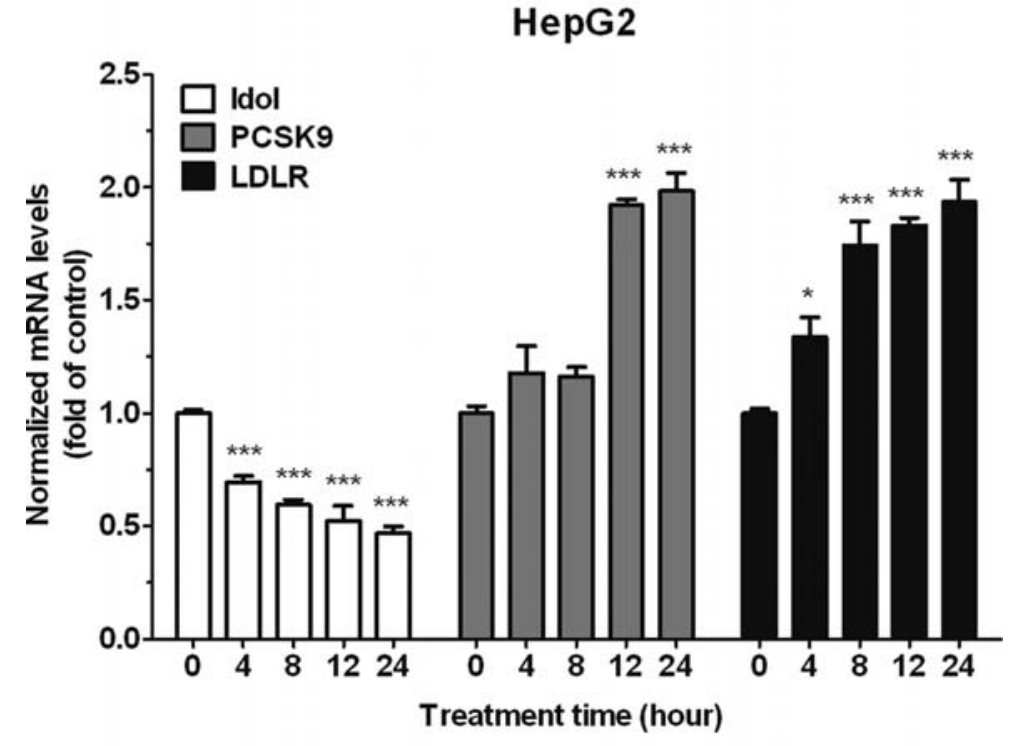

B

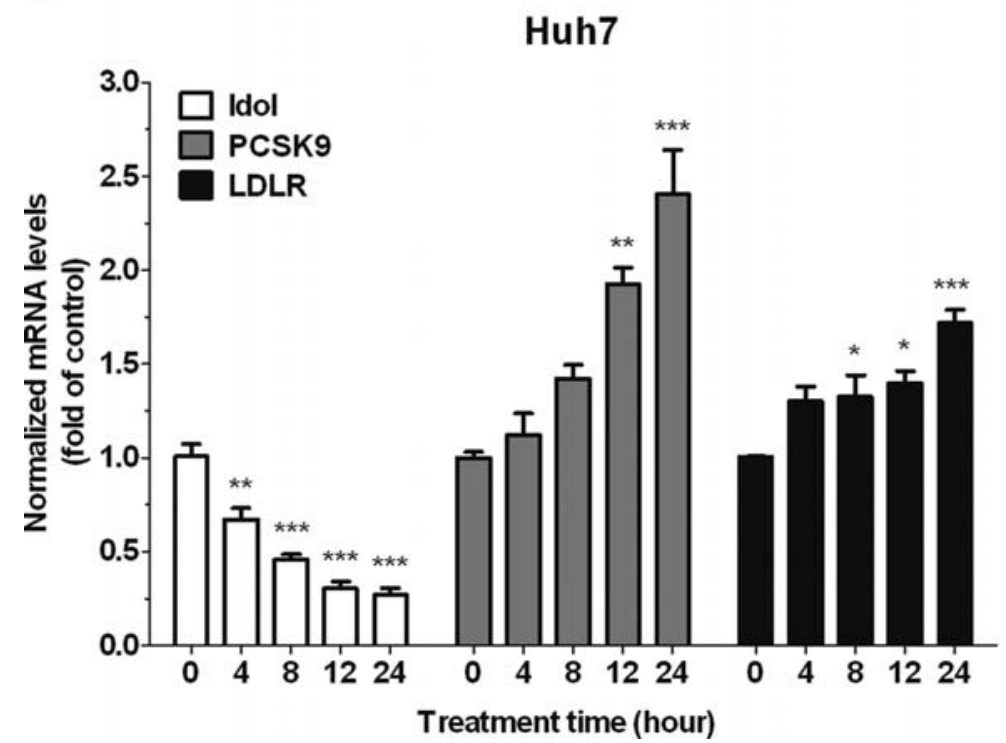

Figure 1. Statin inhibits Idol mRNA expression while up-regulating PCSK9 and LDLR mRNA levels. HepG2 (A) or Huh7 cells (B) were exposed to rosuvastatin (RSV) at a concentration of $5 \mu \mathrm{M}$ for various times. Idol, PCSK9, LDLR, and GAPDH mRNA levels were quantified by real-time PCR. The relative levels of mRNA are presented. Triplicate assays were measured per RNA sample. ${ }^{*} \mathrm{p}<0.05,{ }^{* *} \mathrm{p}<0.01$, and ${ }^{* * * *} \mathrm{p}<0.001$ as compared to 0 h. The data shown are representative of 3 separate experiments.

cells was rapidly decreased by RSV treatment. At 4 h, a $31 \%$ reduction was detected $(\mathrm{p}<0.001)$ and the mRNA level of Idol further declined in RSV treated cells down to $46 \%$ of the untreated control by $24 \mathrm{~h}$. Conversely, RSV increased PCSK 9 mRNA and LDLR mRNA by 2 -fold at $24 \mathrm{~h}(\mathrm{p}<0.001)$. This opposite effect of RSV on Idol and PCSK9 mRNA was corroborated in Huh7 cells (Fig. 1B). A greater than 70\% reduction of Idol mRNA in Huh7 cells was observed after the RSV treatment of $24 \mathrm{~h}$.

To determine whether RSV regulates the gene expression of Idol and PCSK9 with similar potency, we treated cells with various concentrations of RSV for $24 \mathrm{~h}$. RSV at a dose of $1 \mu \mathrm{M}$ effectively suppressed Idol while up-regulating PCSK9 and LDLR mRNA expression in both HepG2 (Fig. 2A) and Huh7 cells (Fig. 2B). We further examined the regulation of
Idol by RSV in primary hepatocyes. Idol mRNA levels in primary hamster (Fig. 2C) and rat (Fig. 2D) hepatocytes declined in a dose-dependent manner after a $24 \mathrm{~h}$ treatment, while PCSK9 and LDLR mRNA levels were increased. The inhibitory effect of statins on Idol gene expression in hamster hepatocytes was further attested by using another HMG CoA reductase inhibitor ATV (Fig. 2E). Cumulatively, these results provided the first evidence demonstrating that statins exert opposite effects on the mRNA expression of Idol and PCSK9 with suppression of the former and induction of the latter.

Concomitant induction of Idol and PCSK9 gene expression by LXR activation. It was shown that activation of LXR by its synthetic ligand GW3965 strongly increased Idol mRNA 
A

HepG2

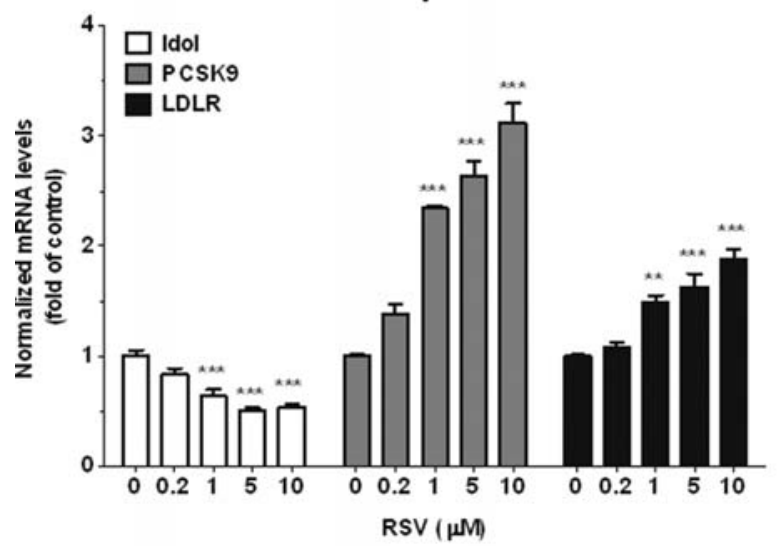

C

Primary Hamster Hepatocytes

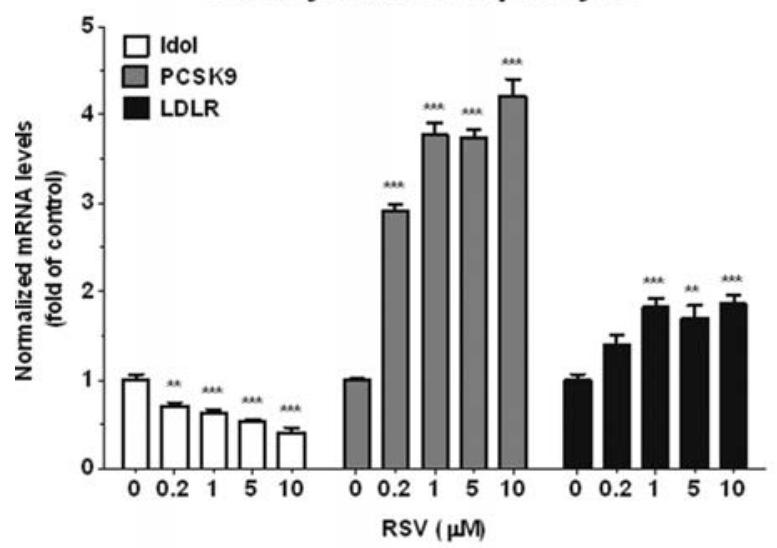

E

Primary Hamster Hepatocytes

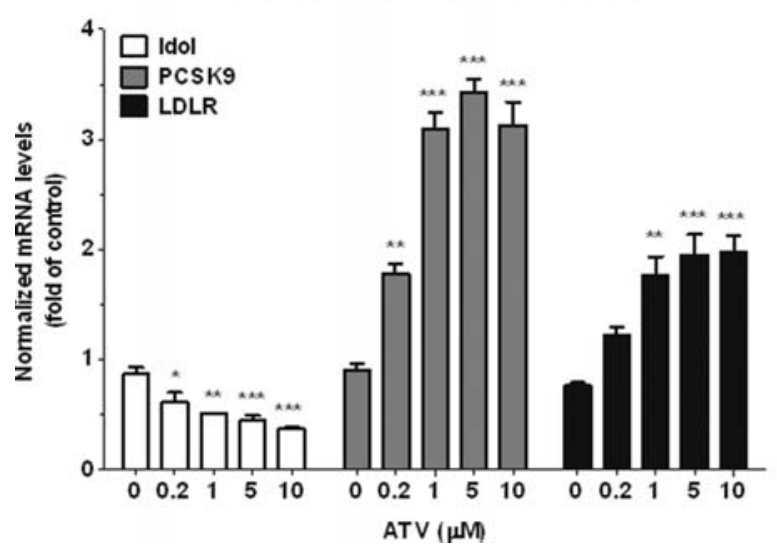

expression in primary mouse hepatocytes and HepG2 cells (17), while the effect of GW3965 on PCSK9 expression was not reported. Since our data showed that activation of the SREBP pathway by statins led to the converse effects on Idol and PCSK9, we sought to investigate the downstream effects of activating the LXR signaling pathways on Idol and PCSK9. We treated HepG2 and Huh7 cells with different doses of GW3965 for $24 \mathrm{~h}$ and measured the Idol, PCSK9, and LDLR mRNA levels by RT-PCR (Fig. 3A and B). At $1 \mu \mathrm{M}$
B

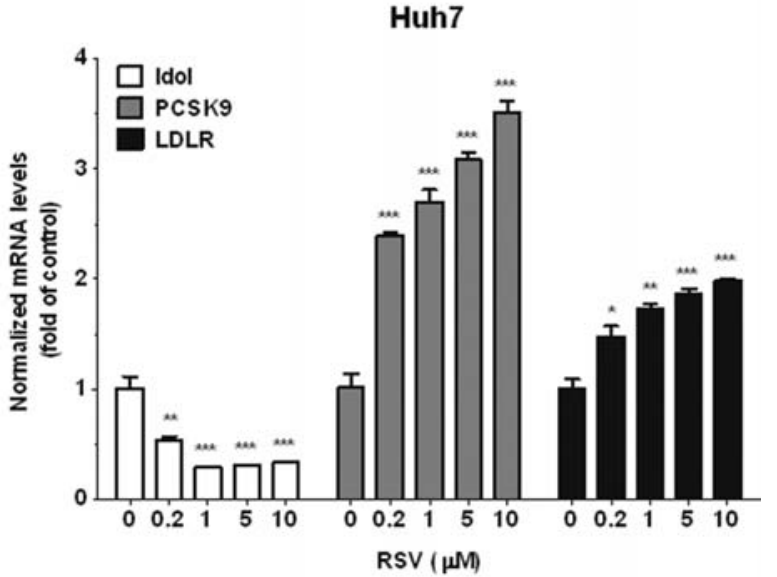

D

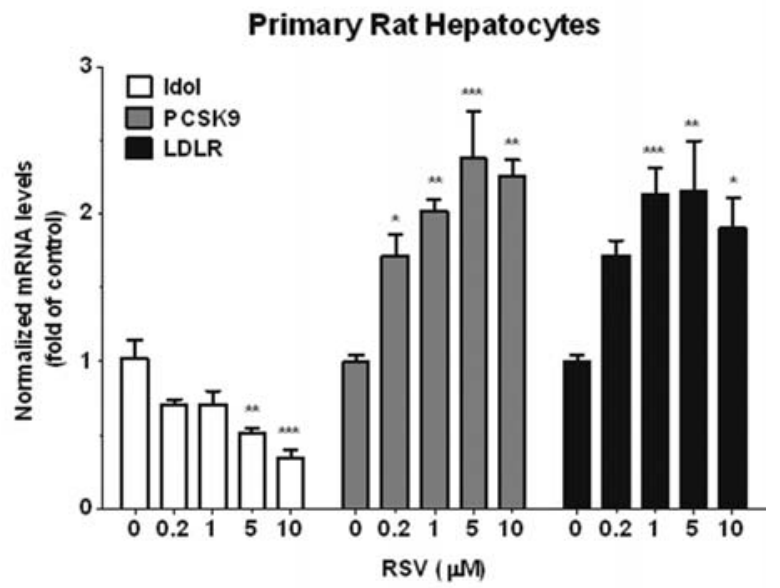

Figure 2. Dose-dependent inhibition of Idol mRNA expression by RSV. HepG2 (A) and Huh7 (B) cells were cultured in LPDS medium overnight. $\mathrm{RSV}$ at indicated concentrations was added to the cells for $24 \mathrm{~h}$ before the isolation of total RNA. Idol mRNA abundance was assayed by real-time PCR along with PCSK9, LDLR, and GAPDH mRNAs. Primary hamster hepatocytes (C and E) and primary rat hepatocytes (D) were isolated and plated in culture plates overnight before the drug treatment. ${ }^{*} \mathrm{p}<0.05$, ${ }^{* *} \mathrm{p}<0.01$, and ${ }^{* * *} \mathrm{p}<0.001$ as compared to untreated control cells. The data shown are representative of 3 separate experiments. concentration, GW3965 increased PCSK9 mRNA levels 1.8and 2.4-fold ( $\mathrm{p}<0.001$ for both) in HepG2 and Huh7 cells, respectively. In both cells lines, PCSK9 mRNA levels were increased over 3-fold by the LXR ligand at a concentration of $5 \mu \mathrm{M}$. In HepG2 cells, GW3965 exerted a stronger effect on Idol than on PCSK9 mRNA, while in Huh7 cells the GW3965-induced increase in PCSK9 mRNA level was comparable to that in Idol mRNA. Activation of the LXR by GW3965 moderately increased LDLR mRNA expression in 
A

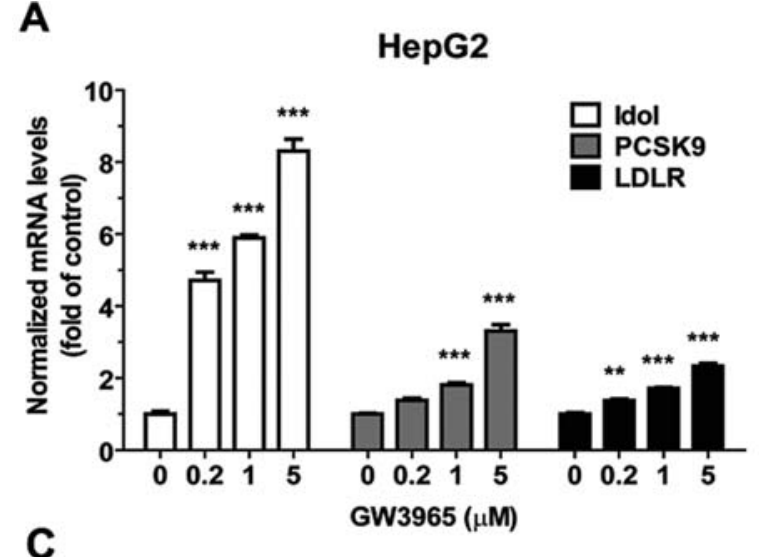

C

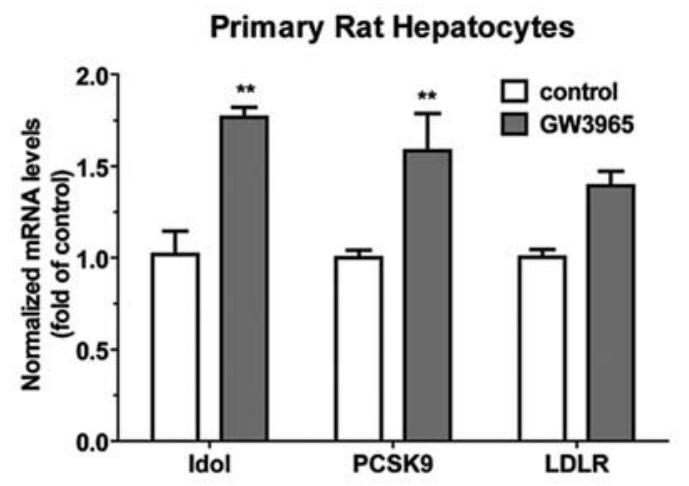

B

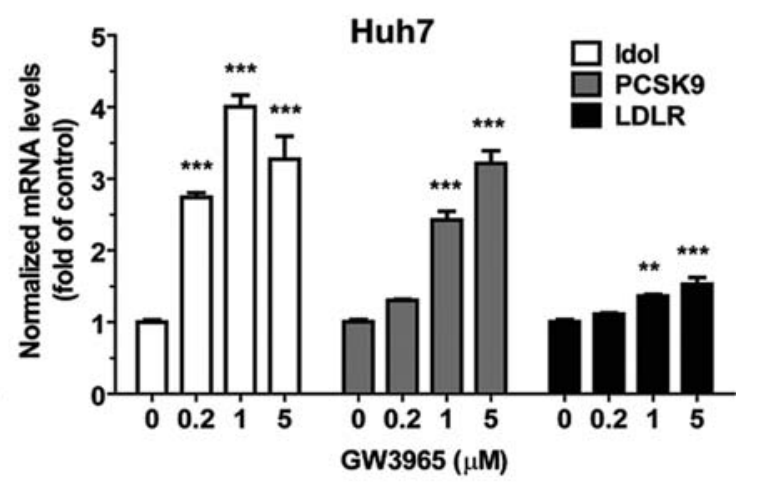

Figure 3. Concomitant increases in Idol and PCSK9 expression by GW3965 treatment lead to a strong reduction of LDLR protein in liver cells. HepG2 (A) and Huh7 (B) cells were treated with various doses of GW3965 for $24 \mathrm{~h}$ prior to the isolation of total RNA. Idol, PCSK9, LDLR mRNA levels were analyzed by real-time PCR and were normalized to GAPDH. Primary rat hepatocytes (C) were cultured in HepatoZYME-SFM medium overnight. GW3965 at $1 \mu \mathrm{M}$ concentration was added to the cells for $24 \mathrm{~h}$ before the isolation of total RNA. Idol mRNA abundance was assayed by real-time PCR along with PCSK9, LDLR, and GAPDH. HepG2 and Huh7 cells were treated with various doses of GW3965 (C) or RSV (D) for $24 \mathrm{~h}$ prior to the isolation of total cell lysate for Western blot analysis of PCSK9, LDLR, and ß-actin.

D

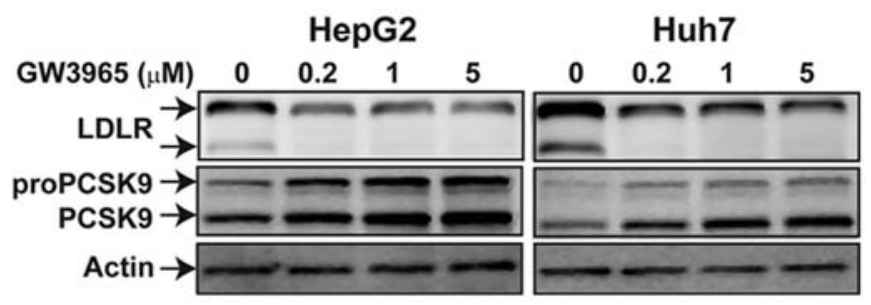

E

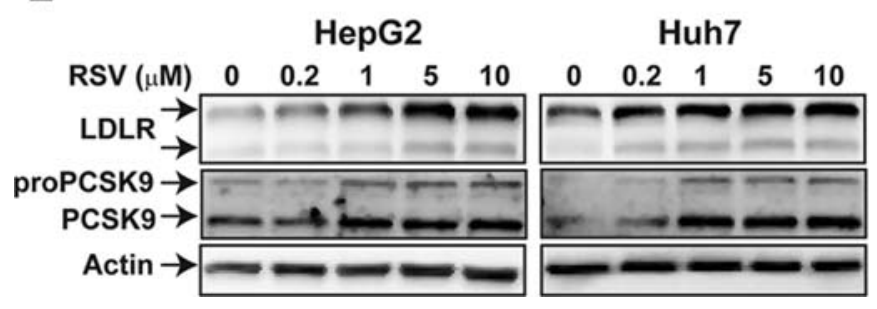

HepG2 cells. The inducing effect of GW3965 on Idol and PCSK9 mRNA expression was further corroborated in primary rat hepatocytes (Fig. 3C).

Next, we compared the effects of the LXR ligand with those of RSV on the protein expression of Idol, PCSK9, and LDLR in HepG2 and Huh7 cells. A dose-dependent increase in PCSK9 protein abundance was observed in GW3965treated cells, confirming its inductive effect on PCSK9 mRNA expression. Despite a GW3965-induced moderate increase in LDLR mRNA levels, the LDLR protein levels were greatly reduced even at the lowest GW3965 dose applied
(Fig. 3D). In contrast to the LXR agonist, RSV, dosedependently increased the amounts of LDLR and PCSK9 in both cell lines (Fig. 3E). Unfortunately, we could not detect Idol protein expression by Western blot in either cell line using two different commercially obtained anti-Idol antibodies. The opposite effects of RSV and GW3965 on Idol mRNA expression could account, at least in part, for the opposite effects of these two compounds, a GW3965-induced increase vs. an RSV-induced decrease, on LDLR protein levels. Altogether, these results demonstrate that activation of the LXR signaling pathway strongly reduces LDLR protein 
A
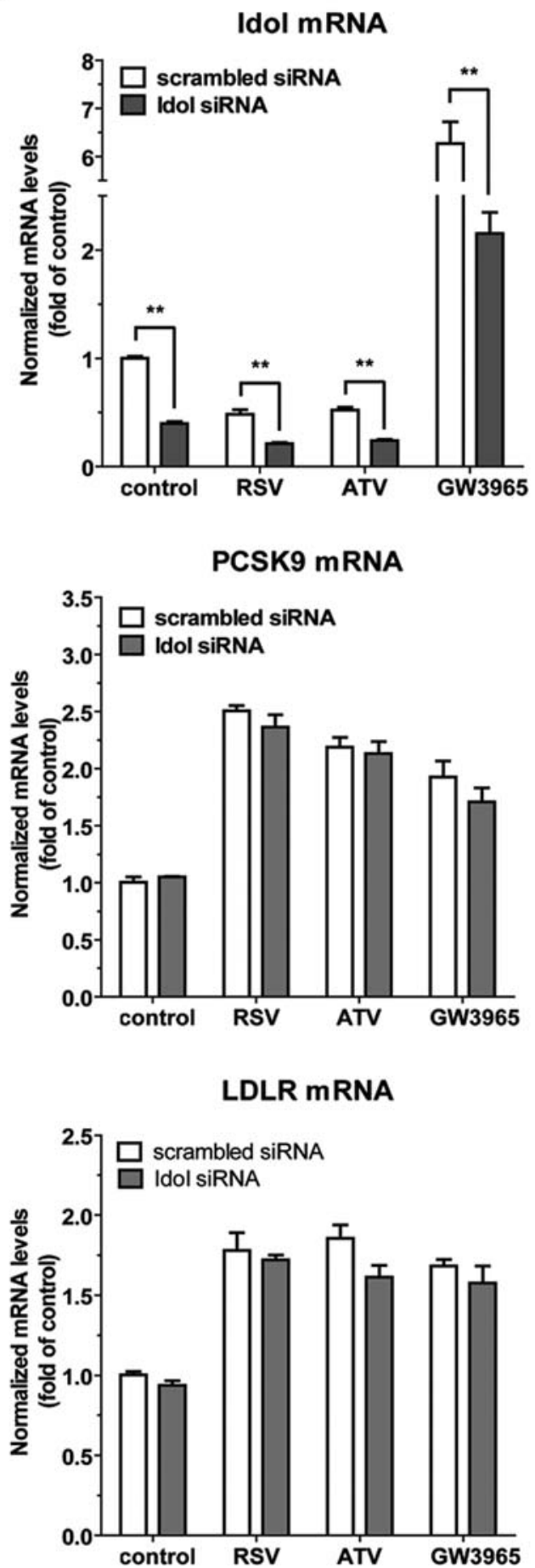

B

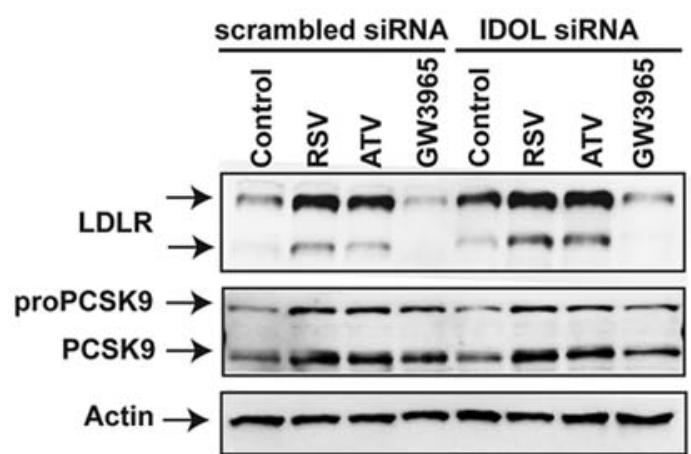

LDLR protein

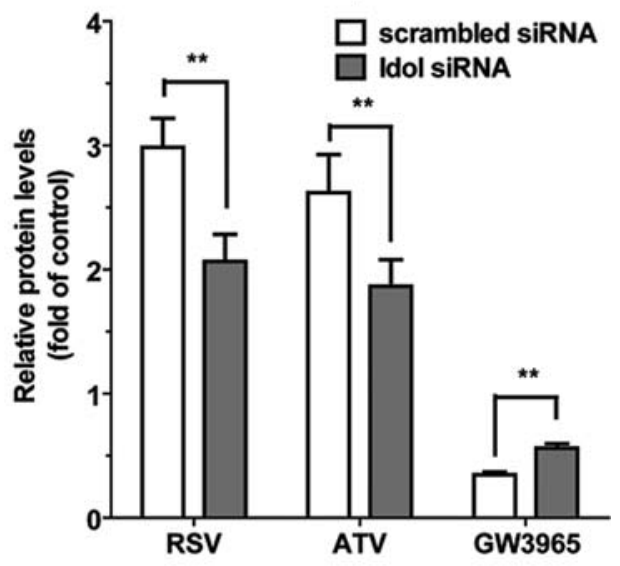

PCSK9 protein

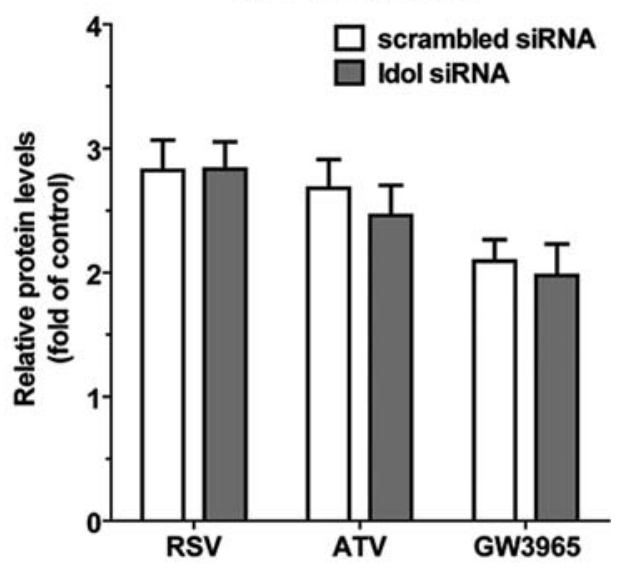

Figure 4. Idol siRNA transfection affected LDLR protein levels in cells untreated and treated with statins or GW3965. HepG2 cells were transfected with Idol siRNA or a control nonspecific siRNA with a scrambled sequence in a culture medium containing $10 \%$ FBS. After 1 day of transfection, cells were cultured overnight in a medium containing $10 \%$ LPDS prior to the treatment with RSV, ATV, or GW3965 at a dose of $5 \mu \mathrm{M}$ for $24 \mathrm{~h}$. At the end of the treatment, total RNA was isolated from transfected cells for real-time PCR analysis of target genes (A), and total cell lysates were isolated for Western blot analysis for LDLR, PCSK9, and ß-actin (B). The LDLR and PCSK9 bands were quantified using an imaging program of Kodak Image Station 4000R. Values were normalized to $\beta$-actin and were graphed relative to untreated cells. The data are presented as mean \pm SEM from results of 4 separate transfection experiments. ${ }^{*} \mathrm{p}<0.05,{ }^{* *} \mathrm{p}<0.01$ as compared to untreated cells.

levels in liver cells through a concomitant up-regulation of Idol and PCSK9, which is in contrast to the activation of the SREBP-signaling pathway that leads to increased LDLR protein expression in liver cells.
Suppression of Idol expression is one underling mechanism for statin-induced up-regulation of LDLR protein expression. Having established that statins inhibit Idol gene expression, we examined the direct impact of Idol depletion on LDLR protein abundance in untreated and statin-treated cells by 
transfecting HepG2 cells with a pool of 4 siRNAs targeting different coding regions of Idol. In untreated control cells, the mRNA level of Idol was reduced by $61 \%(\mathrm{p}<0.01)$ in Idol siRNA transfectants as compared to cells transfected with a scrambled control siRNA (Fig. 4A, upper panel). The expression of Idol mRNA in the specific siRNA transfected cells still responded to LXR ligand or statin treatment although to a lesser extent as compared to cells transfected with the control siRNA. The Idol siRNA transfection had no effect on PCSK9 mRNA (Fig. 4A, middle panel) or LDLR mRNA expression (Fig. 4A, lower panel), demonstrating a gene-targeting specificity of the Idol siRNA. Fig. 4B shows that depletion of Idol did not change PCSK9 protein levels in control or treated cells. However, the basal level of LDLR protein was increased by $60 \%(\mathrm{p}<0.01)$ in Idol siRNA transfected cells compared to that of nonspecific siRNA. Furthermore, increases in LDLR protein abundance by RSV and ATV treatments were tempered by Idol siRNA transfection. The summarized results of densitometry analysis of protein abundances of LDLR and PCSK9 from 4 separate transfection experiments are presented in the middle and lower panels of Fig. 4B. RSV treatment increased LDLR protein levels by 3 -fold in control siRNA-transfected cells. This induction was reduced to 2-fold in Idol siRNA transfected cells. The decrease in RSV enhancing activity on the LDLR protein abundance by Idol depletion was statistically significant $(\mathrm{p}<0.01)$. Idol siRNA transfection caused a similar compromising effect on the ATV-induced up-regulation of the LDLR protein expression (2.6-fold in control siRNA vs. 1.8 -fold in Idol siRNA, $\mathrm{p}<0.01)$. As expected, the inhibitory effect of GW3965 on LDLR protein was partially reversed by the gene-specific siRNA transfection. In contrast to changes in LDLR, depletion of Idol did not affect PCSK9 expression induced by statins or GW3965, suggesting that regulation of LDLR protein stability by Idol is independent of the action of PCSK9.

\section{Discussion}

Idol is a newly identified regulator of the LDLR protein (17). As a ubiquitin ligase, Idol triggers ubiquitination of the LDLR on its cytoplasmic domain, leading to an enhanced degradation of the receptor in the lysosome. Thus, along with the previously characterized PCSK9, LDLR protein stability is modulated by two distinct proteins that are downstream effectors of two major signaling pathways in cholesterol homeostasis, namely the SREBP pathway and the LXR pathway. While it is well documented that the activation of SREBP pathway by statins leads to an increased transcription of PCSK9 concomitant with LDLR, it was unknown until this study that Idol gene expression is also regulated by statins. In this report we have demonstrated that statins reduce Idol mRNA levels while increasing the cellular abundance of PCSK9, a member of the proprotein convertase family.

The inhibitory effect of statins on Idol mRNA expression was consistently observed in all tested liver cells including two hepatoma-derived cell lines and primary hepatocytes isolated from two different rodent species. The statin effect on Idol expression occurred more rapidly than the induction of PCSK9. A significant reduction of Idol mRNA level was detected at $4 \mathrm{~h}$ when PCSK9 mRNA level had not changed. Different mRNA half-lives of Idol and PCSK9 could explain the dissimilar kinetics in response to statin treatment. Our dose-dependent study showed that statins regulate Idol and PCSK9 with comparable potencies. At $1 \mu \mathrm{M}$ concentration, RSV effectively lowered Idol mRNA abundance and raised PCSK9 mRNA levels.

The inhibitory effect of statins on Idol protein expression was not presented in this current study, because we could not detect Idol protein by Western blot analysis after trying all commercially obtainable anti-Idol antibodies. The trouble in detecting endogenous Idol protein has been reported by another study (22). The labile nature of Idol protein was assumed to be responsible for this difficulty. To circumvent this problem and to seek further evidence to support the involvement of Idol in statin-mediated up-regulation of LDLR protein expression we utilized small RNA interference technology to examine the relationship between Idol and LDLR. In untreated cells, transfection of Idol siRNA into HepG2 cells resulted in a $60 \%$ increase $(\mathrm{p}<0.001)$ in LDLR protein levels in the absence of changes in the expression of LDLR mRNA, PCSK9 mRNA, and PCSK9 protein. While LDLR protein abundance was further increased by statin treatment in Idol siRNA transfected cells, there was a statistically significant $(\mathrm{p}<0.01)$ decrease induced by RSV (3-fold control vs. 2-fold specific) or ATV (2.6-fold control vs. 1.8-fold specific). This finding suggests that inhibition of Idol-mediated degradation of the LDLR protein is an integral part of the statin action on LDLR protein expression in addition to its transcriptional effects on LDLR and PCSK9.

The mechanism of statin-induced inhibition of Idol gene expression was not investigated in the current study. Statins inhibit the HMG CoA reductase that leads to the deficiency in oxysterols, the endogenous ligands of LXR. The reduction of the intracellular ligand pool diminishes the transactivating activity of LXRs, thereby impairing the LXR signaling transduction $(23,24)$. Since an LXR response element has been identified in the mouse Idol promoter and this regulatory sequence is required for the induction of Idol promoter activity by GW3965 and LXR $\alpha$ in HEK293 cells (17), it is plausible that the attenuation of LXR transactivating activity by statin treatment led to the reduced expression of Idol in liver cells. Further studies are needed to fully elucidate the underlying mechanism of statin-mediated suppression of Idol gene expression.

In this study, we have also examined the downstream effect of LXR activation on the expression of Idol and PCSK9 in liver cells. Previous studies conducted in cell culture and animal models have produced conflicting results on PCSK9 regulation by the LXR agonists. One study reported that PCSK9 transcription was insensitive to LXR activation in HepG2 cells (12). On the contrary, another study of gene expression profiling detected an inducing effect of the LXR agonist TO901317 on PCSK9 mRNA expression in the mouse liver (19). In the current study, by utilizing the LXR agonist GW3965 we show that LXR activation led to a dosedependent increase of PCSK9 mRNA and protein and Idol mRNA levels (Fig. 3A-C). Furthermore, we detected a strong reduction in LDLR protein levels in GW3965-treated cells, 
which was only partially reversed by the knockdown of Idol. Thus, we speculate that the concomitant increase of Idol and PCSK9 led to an accelerated degradation of the LDLR protein by LXR activation. This inhibitory effect of the ligand on LDLR protein expression observed in HepG2 and Huh7 was different from its in vivo effect. Administration of GW3965 in mice reduced LDLR protein abundance in peritoneal macrophages, the small intestine, but not in the liver (17). The discrepancy between our results and those of the previous report could reflect the differences in the in vitro vs. in vivo study systems. Alternatively, it could be that the liver concentration of GW3965 was not high enough to elicit a response.

In conclusion, we have demonstrated that statins exert opposite effects on the expression of PCSK9 and Idol, the two degraders of the LDLR protein. While PCSK9 induction is a limitation to the clinical efficacy of statins in further lowering cholesterol, inhibition of Idol expression in the liver may contribute to the hypocholesterolemic effects of statins observed in clinical settings. Our new findings suggest that the regulation of hepatic LDLR expression by statins is multifactorial including transcriptional activation through SREBP2 and post-transcriptional modulations by PCSK9 and Idol.

\section{Acknowledgements}

The study was supported by the Department of Veterans Affairs (Office of Research and Development, Medical Research Service) and by grants (1RO1 AT002543-01A1, 1R21AT003195-01A2) from the National Center for Complementary and Alterative Medicine.

\section{References}

1. Nakajima K, Nakajima Y, Takeichi S and Fujita MQ: Plasma reminant-like lipoprotein particles or LDL-C as major pathologic factors in sudden cardiac death cases. Atherosclerosis 198: 237-246, 2008.

2. Homma Y: Predictors of atherosclerosis. J Atheroscler Thromb 11: 265-270, 2004.

3. Zambon A, Brown BG, Deeb SS and Brunzell JD: Genetics of apolipoprotein $\mathrm{B}$ and apolipoprotein AI and premature coronary artery disease. J Intern Med 259: 473-480, 2006.

4. Steinberg D and Witztum JL: Inhibition of PCSK9: A powerful weapon for achieving ideal LDL cholesterol levels. Proc Natl Acad Sci USA 106: 9546-9547, 2009.

5. Brown MS and Goldstein JL: A receptor-mediated pathway for cholesterol homeostasis. Science 232: 34-47, 1986.

6. Goldstein JL and Brown MS: Regulation of the mevalonate pathway. Nature 343: 425-430, 1990.

7. Beglova N and Blacklow SC: The LDL receptor: how acid pulls the trigger. Trends Biochem Sci 30: 309-317, 2005.

8. Scharnagl H and März W: New lipid-lowering agents acting on LDL receptors. Curr Top Med Chem 5: 233-242, 2005.
9. Brown MS and Goldstein JL: The SREBP pathway: regulation of cholesterol metabolism by proteolysis of the membrane bound transcription factor. Cell 89: 331-340, 1997.

10. Brown MS and Goldstein JL: A proteolytic pathway that controls the cholesterol content of membranes, cells, and blood. Proc Natl Acad Sci USA 96: 11041-11048, 1999.

11. Horton JD, Goldstein JL and Brown MS: SREBPs: activators of the complete program of cholesterol and fatty acid synthesis in the liver. J Clin Invest 109: 1125-1131, 2002.

12. Dubuc G, Chamberland A, Wassef H, Davignon J, Seidah NG, Bernier L and Prat A: Statins upregulate PCSK9, the gene encoding the proprotein convertase neural apoptosis-regulated convertase-1 implicated in familial hypercholesterolemia. Arterioscler Thromb Vasc Biol 24: 1454-1459, 2004.

13. Jeong HJ, Lee HS, Kim KS, Kim YK, Yoon D and Park SW: Sterol-dependent regulation of proprotein convertase subtilisin/kexin type 9 expression by sterol-regulatory element binding protein-2. J Lipid Res 49: 399-409, 2008.

14. Zhang DW, Lagace TA, Garuti R, Zhao Z, McDonald M, Horton JD, Cohen JC and Hobbs H: Binding of proprotein convertase subtilisin/kexin type 9 to epidermal growth factorlike repeat A of low density lipoprotein receptor decreases receptor recycling and increases degradation. J Biol Chem 282: 18602-18612, 2007.

15. McNutt MC, Kwon HJ, Chen C, Chen JR, Horton JD and Lagace TA: Antagonism of secreted PCSK9 increases low density lipoprotein receptor expression in HepG2 cells. J Biol Chem 284: 10561-10570, 2009.

16. Cao G, Qian YW, Kowala MC and Konrad RJ: Further LDL cholesterol lowering through targeting PCSK9 for coronary artery disease. Endocr Metab Immune Disord Drug Targets 8: 238-243, 2008

17. Zelcer N, Hong C, Boyadjian R and Tontonoz P: LXR regulates cholesterol uptake through Idol-dependent ubiquitination of the LDL receptor. Science 325: 100-104, 2009.

18. Li H, Dong B, Park SW, Lee HS, Chen W and Liu J: HNF1a plays a critical role in PCSK9 gene transcription and regulation by a natural hypocholesterolemic compound berberine. J Biol Chem 284: 28885-28895, 2009.

19. Repa JJ and Mangelsdorf DJ: The role of orphan nuclear receptors in the regulation of cholesterol homeostasis. Annu Rev Cell Dev Biol 16: 459-481, 2000.

20. Maxwell KN, Soccio RE, Duncan EM, Sehayek E and Breslow JL: Novel putative SREBP and LXR target genes identified by microarray analysis in liver of cholesterol-fed mice. J Lipid Res 44: 2109-2119, 2003.

21. Taghibiglou C, Rashid-Kolvear F, van Iderstine SC, Aiton A, Cavallo D, Cheung $\mathrm{R}$ and Adeli K: Intracellular mechanisms regulating apoB-containing lipoprotein assembly and secretion in primary hepatocytes. J Lipid Res 41: 499-513, 2000.

22. Hong C, Duit S, Jalonen P, Out R, Scheer L, Sorrentino V, Boyadjian R, Rodenburg KC, Foley E, Korhonen L, Lindholm D, Nimpf J, van Berkel TJ, Tontonoz P and Zelcer N: The E3ubiquitin ligase Idol induces the degradation of the low-density lipoprotein receptor family members VLDLR and APOER2. J Biol Chem 285: 19720-19726, 2010.

23. Forman BM, Ruan B and Chen J: The orphan nuclear receptor $\mathrm{LXRb}$ is positive and negative regulated by distinct products of mevalonate metabolism. Proc Natl Acad Sci USA 94: 10588-10593, 1997.

24. Bettowski J: Statins and ALS: the possible role of impaired LXR signaling. Med Sci Monit 16: RA73-RA78, 2010. 